\title{
The Application of a New Wireless RFID Sensing Equipment in Stocktaking
}

\author{
Guicheng Shen, Chaofan Su, Menkang Qian \\ Department of Logistics Engineering, Faculty of information, Beijing Wuzi University, Beijing, China
}

Email address:

874755291@qq.com (Chaofan Su)

\section{To cite this article:}

Guicheng Shen, Chaofan Su, Menkang Qian. The Application of a New Wireless RFID Sensing Equipment in Stocktaking. American Journal of Remote Sensing. Vol. 4, No. 4, 2016, pp. 19-22. doi: 10.11648/j.ajrs.20160404.11

Received: July 10, 2016; Accepted: July 18, 2016; Published: July 29, 2016

\begin{abstract}
In order to solve the problem of manual operation, low efficiency, high error rate in the process of traditional stocktaking, this paper proposes a counting scheme of storage based on a new wireless RFID sensing equipment. By applying the wireless RFID sensing equipment in the stocktaking, it can solve the problem of processing large quantities of data, improve counting efficiency and accuracy, realize the full automatic counting without paper, save manpower and optimize warehouse management.
\end{abstract}

Keyword: Stocktaking, Count, Wireless RFID Sensing Equipment, Efficiency, Accuracy

\section{Introduction}

There are mainly three operations in the traditional warehouse management process, such as stock-in, stocktaking and stock-out. Stock-in means carrying the goods into the warehouse, and gathering the information of the goods. It is hard to collect the precise goods information. Stock-out means carrying goods out of the warehouse, and gathering information of the goods. It has the same problem as the stock-out process. Stocktaking means collecting the information of the specified goods in the library. According to operations of stocktaking, the inventory manager can figure out the quantity of various goods in stock and make purchase plan on the basis of the statistics. What is more, it can confirm the inbound and outbound goods information, at the meanwhile, improve the accuracy of stock-in and stock-out operations. It is obviously that the efficiency and accuracy of stocktaking affects the operation of the entire warehouse management system directly. Therefore, the stocktaking should be used as an important link in the design of warehouse management system. In the rest part, this paper will analyze the deficiency of the current counting form, and proposes a counting scheme based on wireless sensing equipment.

\section{Research on Stocktaking}

Because stocktaking is about collecting the information of target goods, where to stock the goods information and how we get the goods information is very important to us. Here are several stocktaking programs based on different carriers:

\subsection{No Carrier Stocktaking}

The goods information is recorded directly on the outer packing of the goods, and people collect goods information by writing it down on the paper. But manual stocktaking is prone to errors and the efficiency is very low.

\subsection{Bar Code (QR Code) Stocktaking}

Goods information is stored in the bar code or two-dimensional code label, the goods information is scanned by bar code gun. This kind of stocktaking plan has advantage than the no carrier stocktaking: i. The accuracy of information collection is higher; ii. The efficiency of information collection is higher; iii. It unified the format of the information of goods, which is easy to analyze data. However, there also exists the problem of low efficiency in face of large quantities of goods storage. what's more, paper bar code (QR code) has the characteristics of easily damaged, poor capacity of anti pollution [1], which can led to the failure of information collection. 


\subsection{RFID Tag Stocktaking}

Goods information is stored in the RFID (radio frequency identification) tag in the form of electronic signals. RFID tag itself has the characteristics of large capacity, strong ability of anti pollution, non-contact information collection [2], etc. These characteristics determine that RFID tag stocktaking is better than paper bar code or two-dimensional code stocktaking program [3]. There are a few stocktaking forms based on RFID (as a carrier) as follows:

i. The moving truck stocktaking

The moving truck stocktaking means installing RFID reader on the forklift. It completes stocktaking tasks by moving left and right, up and down. Because the forklift need somebody to operate, this program increases the cost of labor;

ii. The rail stocktaking

It means arranging rails on the goods shelves, and the reader is mounted on rails. The goods information is collected by sliding the RFID reader on the rail. Obviously, this program increases the complexity of the management.;

iii. Fixed stocktaking

It means arranging fixed readers in accordance with the needs of storage areas. The goods information is collected by the fixed readers. Because we must make sure that all the goods information on every shelf can be read, we need a large amount of readers and antennas and this means it costs too much.

iv.Manual stocktaking based on RFID

Manual stocktaking based on RFID refers to that the inventory management system delivers the counting targets information to the hand-held RFID reader and the relevant personnel scans the shelf one by one. This program also has the disadvantage of increasing labor cost.

In summary, RFID tag is a best carrier used to inventory. The operating frequency of RFID is divided into three kinds: low frequency, high frequency and ultra high frequency. The four kinds of inventory program above are using high frequency or ultra high frequency RFID. Using high frequency (or ultra high frequency) RFID will produce large quantities of data, which will bring pressure of data processing to the system, such as labels redundant, redundant reader, wrong reading, reading leak and other issues.

Therefore, this paper proposes a RFID stocktaking program based on a new wireless sensing equipment. This program is primarily applying a new wireless sensing equipment to stocktaking, which can achieve automatic paperless stocktaking, save labors, optimize the warehousing and logistics management, and solve the problem of data processing.

\section{A New RFID Wireless Sensing Equipment and Its Advantages}

\subsection{A New RFID Wireless Sensing Equipment}

This paper presents a stocktaking operating system based on a new RFID wireless sensing equipment. This system consists of RFID wireless sensing equipment, networking gateway and the host computer. The messages in RFID tags can be read by installing the RFID tags and the sensing equipment on the same goods, keeping the tags in the reading range at the same time.

This sensing equipment consists of several parts as follows (as shown in figure 1):

It includes a RFID reader module (low frequency), communication module, power control module, power module, product and fixed housing unit. The RFID reader module includes an antenna, a radio frequency module and a processing module [4]; Communication module can be WiFi module, Zigbee module or Bluetooth module; Fixing units can be magnets, suction cups or screws.

i. Reader module

RFID reader module includes an antenna, a radio frequency module and a processing module, which is mainly responsible for the tag information acquisition [5].

ii. Communication module

This module includes a WiFi module, Zigbee module or Bluetooth module wireless communication module [6]. The module is connected with the RFID reader and it is responsible for data transmission from the reader to the gateway, and then uploads data to the host computer through the gateway.

iii. Power module

This module provides electricity for RFID reader module and communication module.

iv.Power control module

The module has two main functions: stabilizing voltage and controlling the timing of the power switch. By controlling the timing of the power, the Power control module prevents the power module from wasting electricity too much. Opening wireless RFID sensing physical switch in chronological order can solve the problem of limited number of access points to the gateway, namely avoid information transmission congestion. This module will ultimately achieve automatic paperless stocktaking, save manpower and optimize the warehousing and logistics management.

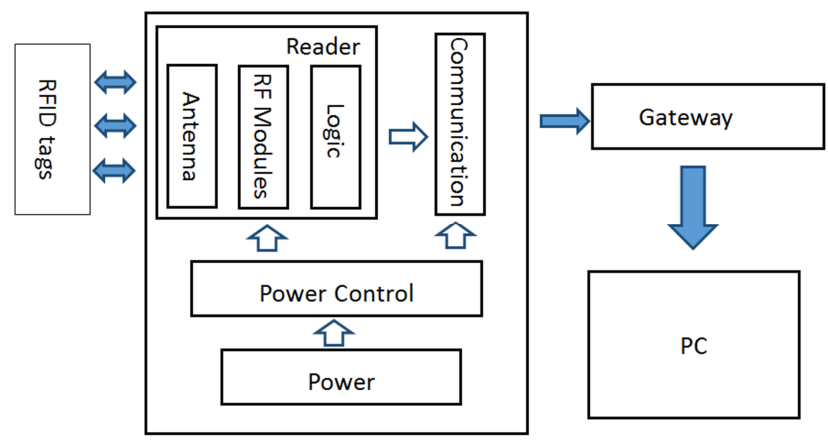

Figure 1. New wireless RFID system structure and its working principle.

\subsection{Advantages}

This system is improved from a traditional RFID system. It has advantages over the original system:

i. Information was transmitted by wireless transmission mode from reader module to $\mathrm{PC}$, which simplifies the management. 
ii. The application of low frequency RFID can improve the accuracy of the information collected and reduce the amount of data.

\section{Stocktaking Classification and Process Design of Stocktaking}

\subsection{Stocktaking Classification}

This article will divide stocktaking into three kinds in accordance with the warehousing processes: Inbound count, Periodic count, Outbound count. Inbound count means counting after the goods entering the warehouse and it is aimed at ensuring the accuracy of the inbound information, the counting result will be added to the inventory immediately; Periodic count means counting goods in the warehouse at intervals of time in accordance with the daily storage management needs. It provides basis for goods purchasing plan and prevents goods from being stolen; Outbound count means counting goods in the warehouse after the goods are shipped out of the warehouse. Its purpose is to ensure the accuracy of the outbound goods information and update inventory information.

\subsection{Process Design of Stocktaking}

Here are process designs for all types of stocktaking:

\subsubsection{Inbound Count}

When goods fitted with electronic tags arrive at warehouse tally district, be unloaded and checked. Then relevant personnel signs for it. The inventory manager uses the forklift truck to carry the goods to pass the Inbound channel, the RFID reader fixed near the channel reads the goods information and matches it with the purchase order. If matched, it produces inbound records, and the inventory management system assigns locations for the goods and plans optimal path.

When the forklift truck arrives the target location, the vehicle mounted RFID reader scans the location RFID tag and binds the location information with the goods to be on the shelves. Then the system deliver a order to the wireless sensing equipment on the goods added just now to make an initialization count. The inventory management system matches the initialization count with the inbound records. If matched, the system updates the inventory information. The specific procedure is as follows (as shown in Figure 2):

i. Forklift truck loads goods and passes through the inbound channel. The RFID reader installed near the inbound channel reads the goods information, then system matches it with the Purchase order information. If not matched, the processing required by the relevant staff, or turn to ii, this process makes sure that the inbound goods information is consistent with the purchase order;

ii. Installing the wireless sensing equipment on goods and turning on the physical switch in turn;

iii. System assigns locations for the goods, then transfers the results to the forklift terminal and guides forklift to the target location according to the planned path [7]; iv. The reader on the forklift reads the location information and binds it with goods information;

v. After the completion of putting goods on the shelves, the host computer sends counting order to wireless equipment to complete the initial stocktaking;

vi.The system will match the result of initial stocktaking with inbound information, if matched, the system updates the inventory information, or the relevant personnel deal with it. This process makes sure that the inbound goods are under the inventory status and well monitored.

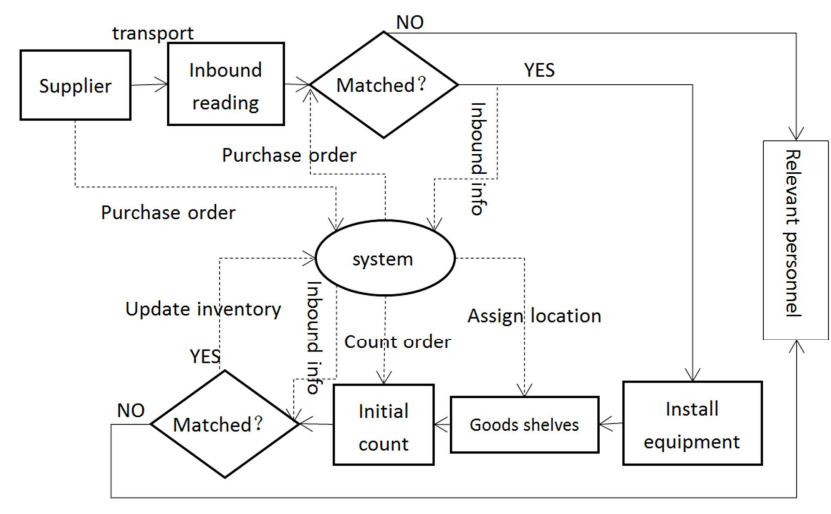

Figure 2. Inbound flow chart.

\subsubsection{Periodic Count}

This part mainly includes counting the goods in warehouse at regular intervals, making purchasing plan and assigning location on the basis of counting result. We can set up the counting cycle for the counting operation. When needed, every counting node is triggered by the system in order to collect the goods information. After a counting, the system matches the counting result with the inventory information, if not matched, the system issued a reminder and the relevant personnel deal with it. At the meanwhile, we can also statistics on the number of different goods. If the number of certain types of goods was lower than the minimum inventory, the system reminds us of timely replenishment.

\subsubsection{Outbound Count}

Warehouse management system automatically determines the optimal delivery mode in accordance with the sales order requirements and generates picking plan. According to the picking plan, the system arranges the sales order picking task and determines the picking area [8]. Then the system reminds staff of picking goods through the forklift terminal, the staff scans tags of goods and location and releases the binding relationship between the two.

When the goods pass through the outlet channel, the reader automatically reads the information of the goods, and transmits the information to the inventory management system. The system compares the goods information with the sales order. If matched, goods can be out of the warehouse and its stock quantity should reduce correspondingly; if not, warehouse management system output prompt message and the relevant personnel deal with it. The specific counting process is as follows (as shown in Figure 3): 
i. The warehouse accepts the sales order, at the meanwhile, the sales order was sent to the warehouse management system and the system makes picking plan on the basis of the sales order [9].

ii. The forklift picks up the goods on the basis of picking plan.

iii. The forklift carries the goods through the outgoing channel, the RFID reader at the outgoing channel reads goods information and matches it with the order. If not matched, the relevant personnel deal with it, or the system finishes the outgoing operation [10]. This process makes sure that the outbound goods information is consistent with the sales order.

iv. The relevant personnel callback the wireless sensing equipment.

v. The system sends counting instructions to the wireless RFID equipment and matches the counting result with the (inventory info - outgoing info), if matched, the system updates the inventory information, or it is handled by the relevant staff. This process makes sure that the rest goods are in right quantity and well monitored.

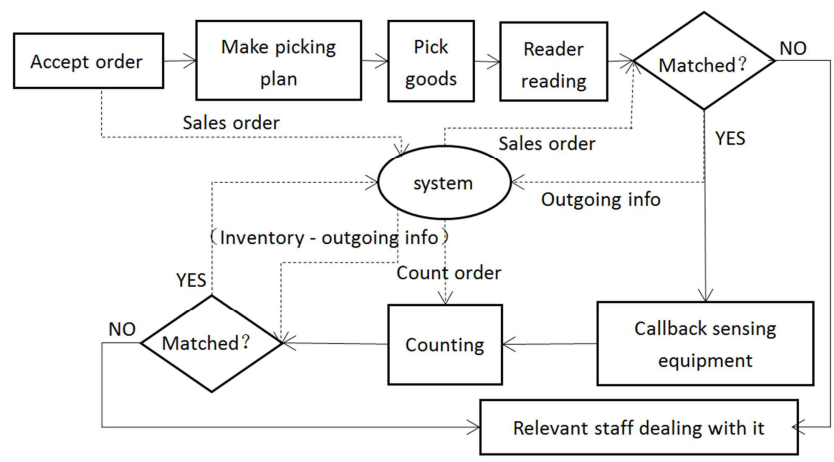

Figure 3. Outbound flow chart brary.

\section{Conclusion}

This paper gives the introduction of a new type of wireless RFID sensing equipment. With the help of the equipment's application in warehouse stocktaking and its advantages in efficiency, accuracy, less data quantity and higher degree of automation, this paper puts forward a counting scheme of storage based on this equipment, namely designing for the process of inbound count, periodic count and outbound count. According to this scheme, it can make sure that the goods information of inbound or outbound can be collected precisely and updated to the system immediately. However, in the face of one (wireless RFID sensing equipment) on one (RFID label) of the information collection, the cost of the program is high. Therefore, this scheme is suitable for the storage of high value and large volume of goods. To sum up, the application of the new wireless RFID sensing equipment in the stocktaking of inventory enriches the stocktaking methods, at the meanwhile, it has a reference value for the application of this equipment in other areas.

\section{References}

[1] Wenrui Hao The application of RFID technology in warehouse management system [D]. Southwest Jiao Tong University, 2011.

[2] Suyan Cui, Ziping Du. Analysis of the application of RFID storage management [D]. 2012. School of economics and management, Tianjin University of Science and Technology.

[3] Yi Yang. Research and application of RFID technology in logistics warehouse management system [D]. East China University of Science and Technology, 2013.

[4] Zhishu Xu. RFID reader and antenna research [D]. Beijing University of Posts and Telecommunications, 2009.

[5] chao Jin. RFID principles and standards [J]. Wireless Internet technology, 2014, 08: 115.

[6] Yunkuan Pan. Research on the application of wireless sensor network based on ZigBee in environment monitoring system [D]. Nanjing University of Science and Technology, 2010.

[7] Qian Feng. Research on the location assignment and order picking optimization of jiuzhoutong pharmaceutical logistics center [D]. Beijing Jiaotong University, 2014.

[8] Xiaohong Fu. Research on application and Design in Logistics Warehouse Management System [D]. Anhui University of Science \& Technology, 2015.

[9] Fengfei Jin. Optimization Analysis and Design of RFID-based warehouse management system workflow [D]. Harbin University of Commerce, 2014.

[10] He Ban. Research and application of warehouse management system based on RFID Technology [D]. Nanjing University of Science \& Technology, 2015. 